\title{
MiR-218 reverses high invasiveness of glioblastoma cells by targeting the oncogenic transcription factor LEF1
}

\author{
YANWEI LIU ${ }^{1 *}$, WEI YAN $^{1 *}$, WEI ZHANG ${ }^{1 *}$, LINGCHAO CHEN $^{2}$, GAN YOU $^{1}$, ZHAOSHI BAO $^{1}$, \\ YONGZHI WANG ${ }^{1}$, HONGJUN WANG $^{2}$, CHUNSHENG KANG $^{3}$ and TAO JIANG ${ }^{1}$ \\ ${ }^{1}$ Department of Neurosurgery, Beijing Tiantan Hospital, Capital Medical University, Beijing 100050; \\ ${ }^{2}$ Department of Neurosurgery, The Second Affiliated Hospital of Harbin Medical University, \\ Harbin 150086; ${ }^{3}$ Laboratory of Neuro-Oncology, Tianjin Neurological Institute, \\ Tianjin Medical University General Hospital, Tianjin 300052, P.R. China
}

Received April 17,2012; Accepted May 18, 2012

DOI: 10.3892/or.2012.1902

\begin{abstract}
The invasive behavior of glioblastoma multiforme (GBM) cells is one of the most important reasons for the poor prognosis of this cancer. For invasion, tumor cells must acquire an ability to digest the extracellular matrix and infiltrate the normal tissue bordering the tumor. Preventing this by altering effector molecules can significantly improve a patient's prognosis. Accumulating evidence suggests that miRNAs are involved in multiple biological functions, including cell invasion, by altering the expression of multiple target genes. The expression levels of miR-218 correlate with the invasive potential of GBM cells. In this study, we found that miR-218 expression was low in glioma tissues, especially in GBM. The data showed an inverse correlation in $60 \mathrm{GBM}$ tissues between the levels of miR-218 and MMP mRNAs (MMP-2, -7 and -9). Additionally, ectopic expression of miR-218 suppressed the
\end{abstract}

Correspondence to: Dr Tao Jiang, Glioma Center, Department of Neurosurgery, Beijing Tiantan Hospital, No. 6 Tiantan Xili, Dongcheng District, Beijing 100050, P.R. China

E-mail: taojiang1964@yahoo.com.cn

Dr Chunsheng Kang, Laboratory of Neuro-Oncology, Tianjin Neurological Institute, 152 Anshan Road, Heping, Tianjin 300052, P.R. China

E-mail:kang97061@yahoo.com

${ }^{*}$ Contributed equally

Abbreviations: GBM, glioblastoma multiforme; AA, anaplastic astrocyteoma; miRNA, microRNA; LEF1, lymphocyte enhancerbinding factor-1; MMP-9, a member of matrix metalloproteinases family; Wnt, Wnt proteins form a family of highly conserved secreted signaling molecules that regulate cell-to-cell interactions during embryogenesis; IHC, immunohistochemistry; FISH, fluorescence in situ hybridization; qRT-PCR, quantitative real-time polymerase chain reaction

Key words: GBM, miR-218, LEF1, MMP-9, invasion, Wnt pathway invasion of GBM cells whereas inhibition of miR-218 expression enhanced the invasive ability. Numerous members of the MMP family are downstream effectors of the Wnt/LEF1 pathway. Target prediction databases and luciferase data showed that LEF1 is a new direct target of miR-218. Importantly, western blot assays demonstrated that miR-218 can reduce protein levels of LEF1 and MMP-9. We, therefore, hypothesize that miR-218 directly targets LEF1, resulting in reduced synthesis of MMP-9. Results suggest that miR-218 is involved in the invasive behavior of GBM cells and by targeting LEF1 and blocking the invasive axis, miR-218-LEF1-MMPs, it may be useful for developing potential clinical strategies.

\section{Introduction}

GBM (WHO-grade IV) is the most malignant and frequent brain tumor and has the worst prognosis of any cancer. Despite advances in treatment by surgery combined with radiotherapy or chemotherapy, patients with GBM have a mean survival of only 14.6 months (1). Therefore, new therapeutic targets are urgently needed. A formidable difficulty in treating GBM is that tumor cells diffuse and infiltrate the normal peripheral tissue, therefore it is not possible to completely dissect out and remove the tumor. Degradation of extracellular matrix (ECM) is the defining step in tumor cell invasion and members of the matrix metalloproteinase family (MMPs) have crucial roles in regulating this process (2-4). Thus, understanding and blocking the invasive process may be an effective strategy for treating GBM.

The recent discovery of miRNAs is a major advance. Accumulating evidence suggests that miRNAs are involved in multiple biological functions, including cell invasion, by altering the expression of multiple target genes. They bind to the $3^{\prime}$ untranslated region (UTR) of target messenger RNAs (mRNAs) to suppress translation or induce degradation of these mRNAs. The roles different miRNAs play have been recently expounded in numerous human tumors as detailed below.

Accumulated evidence shows that downregulation of miR-218 can enhance tumor cell invasion and proliferation in several kinds of solid tumors $(5,6)$. In this study, we have identified that miR-218 is downregulated in GBM tissues and has a 
considerable involvement in GBM cell invasion. Ectopic expression of miR-218 can decrease the invasive ability of a GBM cell line. Conversely, inhibiting miR-218 expression can increase this ability. Our data shown an inverse correlation in GBM tissue between levels of miR-218 and MMP mRNAs (MMP-2, -7 and -9). These members of the MMP family are downstream effectors of the Wnt pathway $(7,8)$. Using sophisticated algorithms from target prediction databases and in vitro tests, we found that LEF1, a nuclear transducer involved in the Wnt/ $\beta$ catenin pathway, is a candidate target of miR-218. MiR-218 regulates cell invasive ability by targeting LEF1, resulting in reduced synthesis of MMPs. Furthermore, ectopic expression of miR-218 can reduce protein levels of LEF1 and MMP-9 in GBM cell line and inhibiting miR-218 expression can increase their protein expression that was detected by western blotting. More importantly, LEF1 siRNA can imitate the role of miR-218. Luciferase reporter assay further confirmed the direct interaction between miR-218 and the 3' UTR of LEF1 mRNA. Our previous work has shown that MMP-9 is associated with GBM recurrence and poor patient prognosis (9). However, the reason for the increased expression of MMP-9 in GBM tissues remains poorly understood.

Wnts are a family of secreted glycoproteins with diverse roles in tumor development, including regulation of cell invasion. Wnt signaling stabilizes $\beta$-catenin protein and directly targets the MMP promoters (MMP-2, -9 and -7) through the LEF/TCF complex $(7,8)$. Additionally, our previously unpublished data, obtained by miRNA microarray and gene expression profiling, showed that the mRNA levels of MMP-9 and -7 were inversely related to the expression of $\mathrm{miR}-218$ in $60 \mathrm{GBM}$ tissues. These data suggest that upregulation of miR-218 can decrease MMP-7, and-9 expression by targeting LEF1 and may be an efficient strategy in preventing glioma cell invasion.

\section{Materials and methods}

Clinical samples. Tumor specimens (GBM) were obtained from patients who underwent positive debulking surgery in the Neurosurgery Department of Beijing Tiantan Hospital from 2006 to 2009. The diagnosed gliomas were re-reviewed in histological slides by the experiential neuropathologist according to the 2007 WHO classification. Normal brain tissue samples were obtained from internal decompression of patients with cerebral injury and temporal lobe resection for epilepsy. Tissue samples were fixed by formalin, embedded by paraffin and tissue microarray blocks comprising a total of 38 tissue cores (4 normal, 8 low grade, 8 AA and 18 GBM) were constructed with a tissue microarrayer (Beecher Instruments, USA). The tissue microarrays were stored at $4^{\circ} \mathrm{C}$ until analysis for IHC and FISH. The study complied with the requirements of the local ethics committee. Individual informed consent was obtained from all participants.

Cell lines and transfection. The human GBM cell lines U251, U87, SNB19 and LN229, obtained from the Institute of Biochemistry and Cell Biology, Chinese Academy of Science, were used in this study. Cells were maintained in DMEM containing $10 \% \mathrm{FBS}, 50 \mathrm{U} / \mathrm{ml}$ penicillin $\mathrm{G}$, and $250 \mu \mathrm{g} / \mathrm{ml}$ streptomycin in a humidified atmosphere containing $5 \% \mathrm{CO}_{2}$ at $37^{\circ} \mathrm{C}$. Transfections with miR-218 were performed in serum- free medium $24 \mathrm{~h}$ after plating, using Lipofectamine 2000 (Invitrogen). After $6 \mathrm{~h}$, cells were placed in complete medium and maintained at $37^{\circ} \mathrm{C}$ in $5 \% \mathrm{CO}_{2}$. The miR-218 mimic sequence used was 5'-UUGU GCUUGAUCUAACCAUGUAU GGUUAG AUCAAGCACAAUU-3'. Inhibitor sequence: 5'-ACAUGGUUA GAU CAAG CACAA-3', LEF1-1589 siRNA: 5'-CAUCCCGA GAACAUCAAAUTTAUUUGAUGUUCUCGGGAUGTT-3'

(Gima Biol Engineering Inc., Shanghai, China).

Western bloting. Cells were lysed $1 \%$ Nonidet P-40 lysis buffer $48 \mathrm{~h}$ following exposure to LY294002 or vehicle. Homogenates were clarified by centrifugation at $20,000 \mathrm{x}$ g for $15 \mathrm{~min}$ at $4^{\circ} \mathrm{C}$, and protein concentrations were determined with a bicinchoninic acid protein assay kit (Pierce Biotechnology). SDS-PAGE was performed on $40 \mu \mathrm{g}$ of protein from each sample, gels were transfered to PVDF membranes (Millipore) and incubated with primary antibodies detecting LEF1, MMP-9 (Cell Signaling Technology; 1:1000 dilution), TIMP-1 (Santa Cruz; 1:1000 dilution) followed by incubation with an HRP-conjugated secondary antibody (Zymed, San Diego, CA; 1:1000 dilution). The specific protein was detected using a SuperSignal protein detection kit (Pierce, USA). Membranes were stripped and reprobed with a primary antibody against GAPDH (Santa Cruz; 1:1000 dilution).

Immunohistochemistry (IHC) and immunofluorescence (IF). IHC was performed on a glioma tissue array by the avidinbiotin-complex (ABC) method as previously described (10). Briefly, the sections were incubated with primary antibody $(1: 100$ dilution) overnight at $4{ }^{\circ} \mathrm{C}$, then incubated with a biotinylated secondary antibody (1:200 dilution) at room temperature for $1 \mathrm{~h}$, followed by the incubation with ABC-peroxidase reagent (1:200 dilution, Vector, USA) for an additional $1 \mathrm{~h}$. After washing with Tris-buffer, the sections were stained with DAB (3,3 diaminobenzidine, $30 \mathrm{mg}$ dissolved in $100 \mathrm{ml}$ Tris-buffer containing $0.03 \% \mathrm{H}_{2} \mathrm{O}_{2}$ ) for $5 \mathrm{~min}$, rinsed in water and counterstained with hematoxylin. The antibodies used in this study were those to LEF1 and MMP-9 (Cell Signaling Technology). Negative controls were obtained by substituting primary antibodies with non-immune serum. The proportion of positively stained tumor cells was graded as follows: 0, no positive tumor cells; $1,<5 \%$ positive tumor cells; $2,5-20 \%$ positive tumor cells; and $3,>20 \%$ positive tumor cells $(11,12)$. The intensity of staining was recorded on a scale of 0 (no staining), 1 (weak staining, light yellow), 2 (moderate staining, yellowish brown) and 3 (strong staining, brown). The staining index was calculated as follows: staining index $=$ staining intensity $\mathrm{x}$ proportion of positively stained tumor cells.

IF was performed on LN229 cell line as previously described (13). Cells were incubated with LEF1 antibody (1:100 dilution) for $1 \mathrm{~h}$ at room temperature. TRITC-labeled secondary antibodies were added at 1:100 dilution, and the cells were then incubated for another $30 \mathrm{~min}$. Nuclei were stained with 4,6-diamidino2-phenylindole (DAPI; Invitrogen).

Fluorescence in situ hybridization (FISH). FISH was subsequently performed on the same tissue array using a miR-218 Probe Kit (Boster Co., Wuhan, China) according to the manufacturer's instructions. The sequence of miR-218: 5'-ACATGGT TAGATCAAGCACAA-3'. The expression of miR-218 in each 
spot was estimated by an epifluorescence microscope (Olympus, Tokyo, Japan) and graded according to a previous method. In three separate regions in each spot $\geq 100$ cells was calculated. The degree of fluorescence was determined by combining the proportion of positively stained tumor cells and the intensity of staining. The proportion of positively stained tumor cells was graded as follows: $0,<5 \%$ positive tumor cells; $1,5-30 \%$ positive tumor cells; and 2, 30-70\% positive tumor cells. The intensity of staining was recorded on a scale of 0 (no light), 1 (weak light), 2 (moderate light) and 3 (strong light). The staining index was calculated as follows: staining index $=$ staining intensity + proportion of positively stained tumor cells.

Transwells. Transwell filters (Costar, USA) were coated with Matrigel $(3.9 \mu \mathrm{g} / \mu \mathrm{l}, 60-80 \mu \mathrm{l})$ on the upper surface of the polycarbonic membrane (diameter $6.5 \mathrm{~mm}$, pore size $8 \mu \mathrm{m}$ ). Following $30-\mathrm{min}$ incubation at $37^{\circ} \mathrm{C}$, Matrigel solidified and served as the extracellular matrix for tumor cell invasion analysis. Harvested cells $\left(1 \times 10^{5}\right)$ in $100 \mu l$ of serum-free DMEM were added into the upper compartment of the chamber. The experimental procedure was as previously described (13).

Scratch assay. LN229 and U87 cells were grown in 6-well plates with complete medium. After $90 \%$ confluence was reached, the medium was replaced with FBS-free media for $24 \mathrm{~h}$. Wound was created by a germ-free $100 \mu \mathrm{l}$ pipette tip in the monolayer. The cells were washed with PBS and grown in FBS-free media for $36 \mathrm{~h}$. The wounds were observed under a phase contrast microscope (IX81, Olympus). The images were analysed by drawing lines at the wound edges. The width of the scratch was measured at 0 and $36 \mathrm{~h}$ post-treatment. The migration distance in the wound was calculated according to the following formula: cell-free area at $0 \mathrm{~h}$ - cell-free area at $36 \mathrm{~h}$. Experiments were repeated thrice in duplicate with comparable results.

Quantitative real-time polymerase chain reaction ( $q R T-P C R)$. qRT-PCR analysis of miR-218 expression in 4 GBM cell lines was performed as previously described (14). Briefly, total RNA from cells was extracted by TRIzol (Invitrogen) and subjected to reverse transcription using a first-strand cDNA synthesis kit (Invitrogen) according to the manufacturer's instructions. The quantitative analysis of the change in expression levels was calculated by real-time PCR machine (7500 ABI, USA). For detection of miR-218, the TaqMan MicroRNA assay kit (Applied Biosystems) was used according to the manufacturer's instructions. U6 was used as an internal control to normalize variances.

Luciferase assay. Cells were seeded in 96-well plates and culture for $24 \mathrm{~h}$. The reporter plasmid was purchased from GenScript (Jiangsu,China).The3'-UTR binding sequence was subclonedinto a firefly luciferase-based reporter construct immediately downstream of $L u c$ coding sequence. The luciferase reporter plasmids containing wild or mutant 3'-UTRs of LEF1 were transfected into cells together with miR-218 mimics, pGL3-control-wild LEF1: 5'-AAATGTAAAAGCACATGAGAAT-3'; pGL3control-mutant LEF1: 5'-AAAGTACGGATCCGTGAGAAT-3'. The luciferase reporter assay was carried out as previously described. Three independent experiments were performed and the data are presented as means \pm SD.
Statistical analysis. All tests were done using SPSS Graduate Pack 13.0 statistical software (SPSS, Chicago, IL). Descriptive statistics including the mean $\pm S E$ along with one-way ANOVAs were used to determine significant differences. Non-parametric test was performed in the grading system. $\mathrm{P}<0.05$ was considered significant.

\section{Results}

MiR-218 is downregulated in gliomatissues and inversely related to MMP-9 and MMP-7 mRNA expression in GBM tissues. Previous studies have reported that miR-218 is downregulated in cervical cancer, lung cancer and gastric cancer $(5,15,16)$. Similarly, in both AA and GBM, miR-218 is also expressed at a low level, compared to normal brain tissue; $\mathrm{P}=0.009$ or $\mathrm{P} \leq 0.01$ $(17,18)$. To identify whether the expression of miR-218 is downregulated in gliomas of Chinese patients, FISH was performed using a tissue array that included 38 samples (4 normal tissues, 8 low grade, 8 grade III and 18 grade IV samples). Consistent with previous observations, we observed that the expression level of miR-218 was significantly decreased in glioma tissues, especially in grade III/IV tissues $(\mathrm{P}=0.012 / 0.003)$, compared to normal brain tissue (Fig. 1A). Interestingly, this result shows that miR-218 expression decreases markedly from normal brain tissue to low grade to GBM tissue (I/II VS III or IV, $\mathrm{P}=0.021$ or 0.001$)$.

For tumor cell invasion, obvious candidates for signaling molecules are members of the MMP family and the relationship between MMP-9 levels and GBM recurrence over a short time period, or poor patient prognosis was reported in our previous study (9). Our previously unpublished data, obtained by miRNA microarray and mRNA expression profiling, showed that mRNA expression of MMP-9 $(\mathrm{P}=0.001)$ and MMP-7 $(\mathrm{P}=0.002)$ is inversely related to the expression of miR-218 in $60 \mathrm{GBM}$ tissues (Fig. 1B). Given the above data, we suggest that the invasive function of miR-218 may be carried out by MMP-7 and -9. Due to the common function of MMP-9 and -7 and the lack of specific reagents, MMP-7 was not investigated further in this study.

Upregulation of miR-218 inhibits GBM cell invasion and inhibiting miR-218 expression enhanced this ability. The role of miRNAs in tumor cell invasion has been intensively studied in recent years. To identify the invasive function of miR-218, four GBM cell lines (LN229, SNB19, U251 and U87) were screened for miR-218 expression levels by qRT-PCR. U87 cells had the highest level of miR-218 expression, while LN229 cells had the lowest (Fig. 2A). Cells were then assayed in a transwell invasion assay. LN229 and U87 cells transfected with specific miR-218 mimics and inhibitor, respectively. The processed cells were incubated in 6-well plates for $24 \mathrm{~h}$ and then plated on the upper Matrigel plugs of the transwells. The transwell assay showed that overexpression of miR-218 significantly suppressed the invasive ability of LN229 cells by $\sim 3$-4-fold, while inhibiting miR-218 expression enhanced this ability by $\sim 6$-fold $(\mathrm{P}<0.05)$ (Fig. $2 \mathrm{C}$ ). Indeed, a similar effect of miR-218 inhibiting cell invasion and of the miR-218 inhibitor promoting invasion were also observed in the scratch assay (Fig. 2B). Migration ability was inhibited by $\sim 2$-fold by overexpression of miR-218 compared to negative controls. 


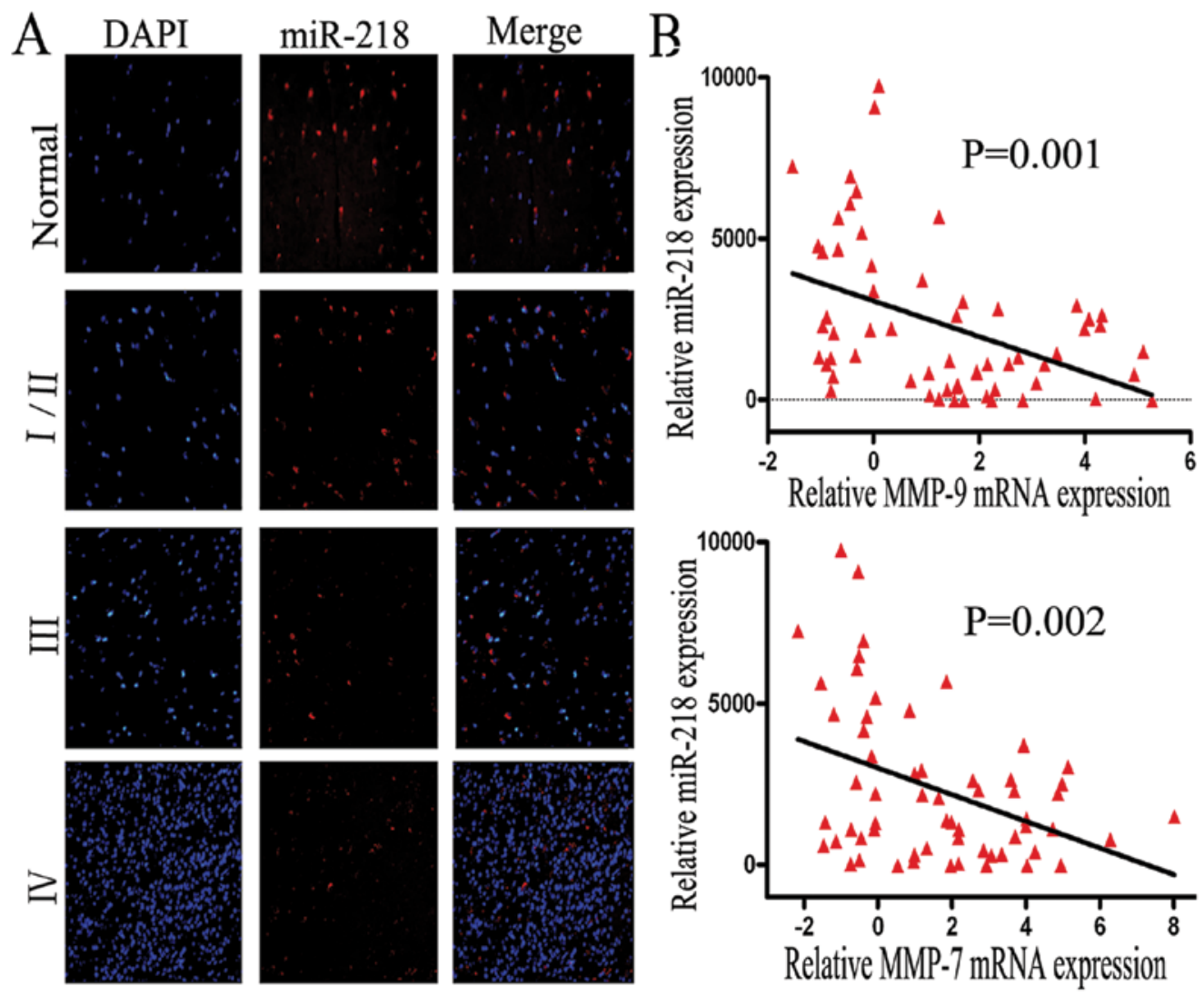

Figure 1. MiR-218 is expressed at low levels in glioma tissue and inversely correlated with the mRNA levels of MMP-9 or MM-7 in 60 GBM tissues. (A) FISH assay to detect miR-218 expression in 38 tissues comprised of 4 normal brain samples, 8 low grade, 8 anaplastic and 18 GBM samples. Reactivities were visualized using the TSA Cy3 system (red), and nuclei were stained with DAPI (blue). (B) The inverse relationship between levels of miR-218 and MMP-9 (P<0.001) and MMP-7 mRNAs $(\mathrm{P}<0.002)$ was detected in $60 \mathrm{GBM}$ tissues.
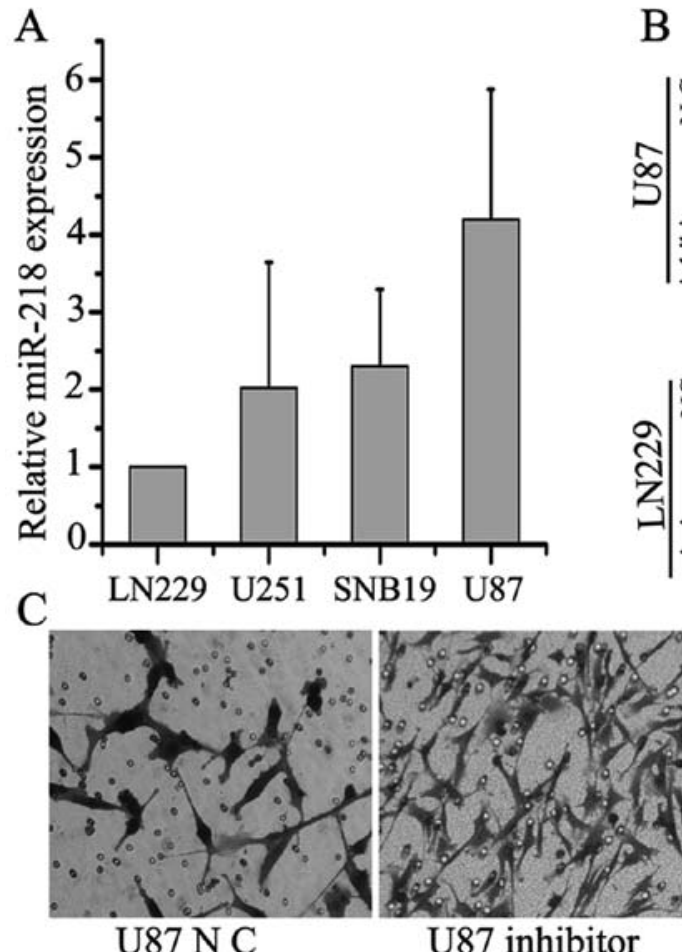

Unhibitor
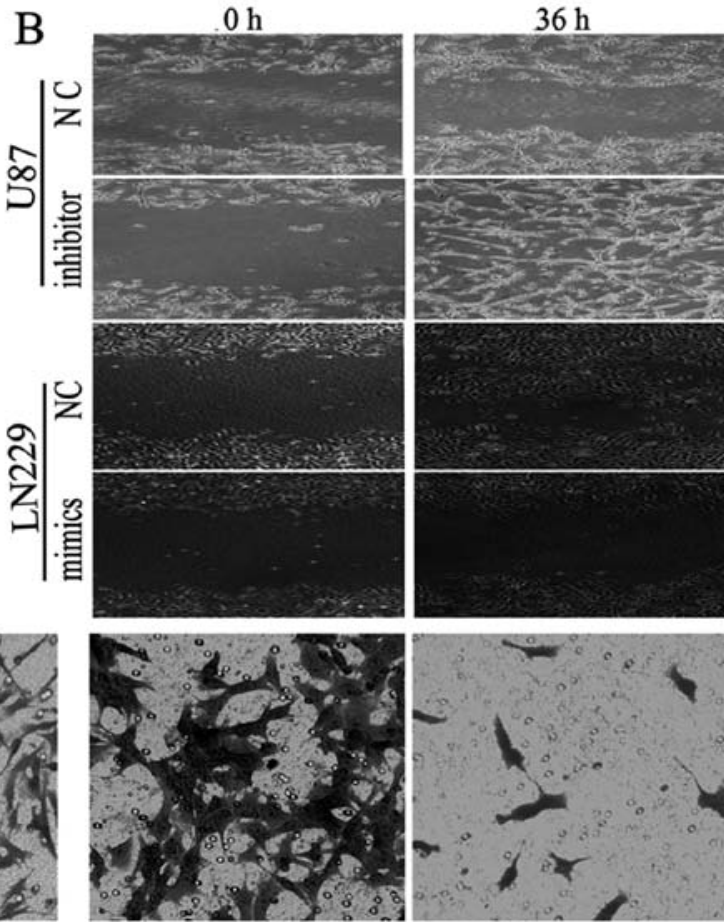

LN229 N C

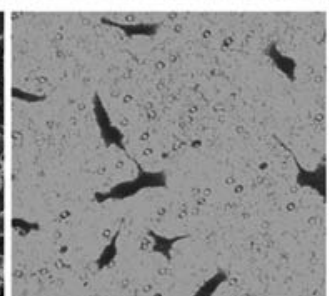

LN229 mimics

Figure 2. MiR-218 can suppress GBM cell invasion. (A) The relative expression levels of miR-218 were detected in four GBM cell lines. (B) Wound healing assay to determine the migratory capacity of miR-218 mimics/inhibitor-transfected LN229/U87 cells. (C) Transwell assay to determine the invasive ability of miR-218 mimics/inhibitor-transfected LN229/U87 cells. Each assay was performed three times in independent experiments. Error bars indicate standard deviation. $\mathrm{P}<0.05$. 
A

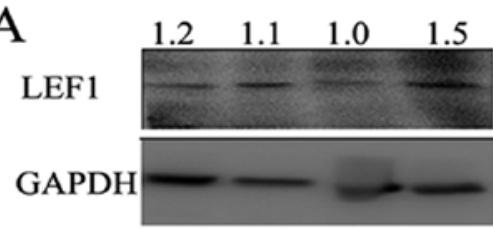

B

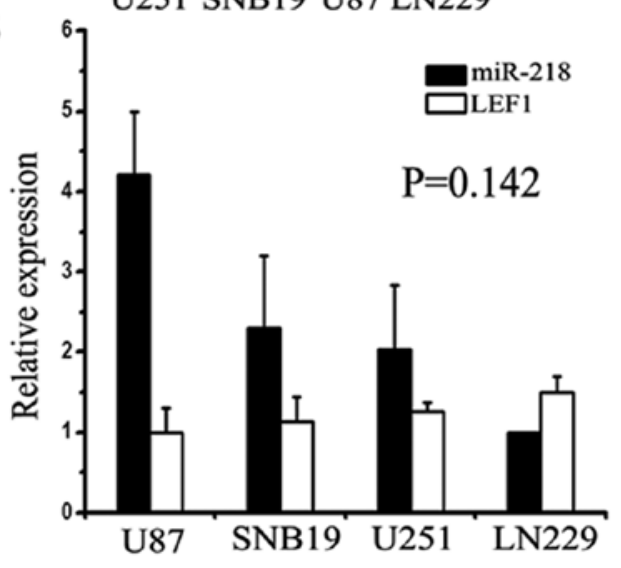

D

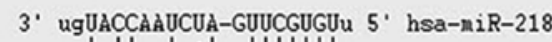
$257: 5^{\circ}$ aaAAGGAAAUGUAAAAGCACAU $3^{\circ}$ LEF1

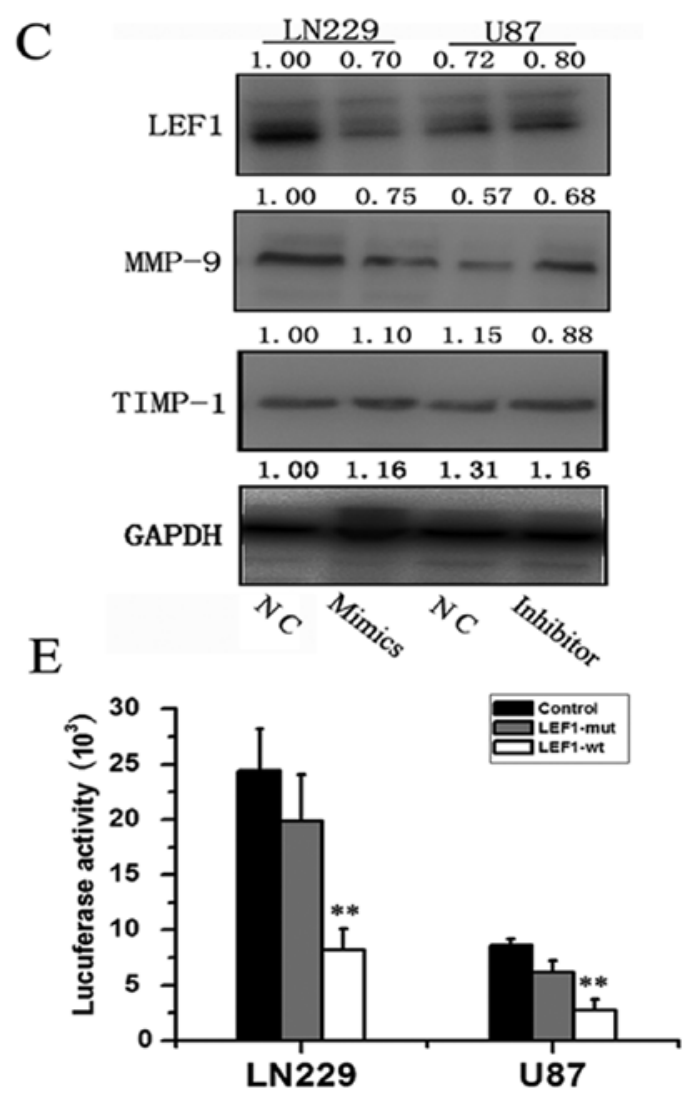

Figure 3. LEF1 is a direct target of miR-218. (A) Western blot analysis and qRT-PCR to detect LEF1 protein expression and miR-218 expression in four GBM cell lines, respectively. (B) The inverse relationship is depicted by the histogram. (C) Western blot analysis to detect LEF1, MMP-9 and TIMP-1 protein levels after exogenous expression of miR-218 mimics/inhibitor in LN229/U87 cell lines. (D) Identification of target sites in the 3'-UTR of LEF1 was performed in silico. (E) Luciferase assay to determine the target relationship in the two cell lines. Luciferase activity was evaluated as described in Materials and methods. Representative of at least three independent experiments. ${ }^{* *} \mathrm{P}<0.01$.

This finding indicates that miR-218 can suppress the invasive ability of GBM cell lines in vitro. However, whether or how the effectors MMP-9 and MMP-7 were regulated by miR-218 remains unclear.

Mature miR-218 directly targets LEF1 and regulates MMP-9 expression. The $\mathrm{Wnt} / \beta$-catenin/LEF1 pathway is one of the best known signaling pathways and it correlates with tumorigenesis, especially with tumor cell invasion and adhesion. Increasing MMP-9 expression is associated with dysfunctions of Wnt signaling (19); therefore, we conceived a biological transport chain: the miR-218-intermediary molecule-MMP-9 axis. MMP-9 is a key molecule downstream of Wnt activation and is responsible for increased rates of neural stem cell (NSC) proliferation and migration in $1 \% \mathrm{O}_{2}$ (19). MMP-7 is also downstream effector in the Wnt pathway; therefore, we hypothesized that miR-218 can regulate the expression of MMP-9 and -7 by targeting transcription factors involved in the Wnt pathway. Using sophisticated algorithms in miRanda (http://www.microrna.org/), we identified LEF1 as a candidate target of miR-218 (Fig. 3D). To validate this, we detected LEF1 protein levels in the same four cell lines and normalized these levels to the level of GAPDH (Fig. 3A). As expected, an inverse relationship between miR-218 and LEF1 protein was confirmed by qRT-PCR and western blot analysis $(\mathrm{P}=0.142)$ (Fig. 3B). Although the P-value was not significant due to the limited number of cell lines, the relationship was quite clear. To explore this functional axis: miR-218-LEF1-MMP-9, we re-expressed miR-218 in LN229 cells by transfection of miR-218 mimics and knocked down miR-218 in U87 cells using its inhibitor. We then detected changes in levels of LEF1 protein. Western blotting confirmed a 1.43-fold decrease in levels of LEF1 $48 \mathrm{~h}$ after re-expression of miR-218 mimics in LN229 cells, whereas significantly increased expression was observed $48 \mathrm{~h}$ after transfection of miR-218 inhibitor compared to negative controls (Fig. 3C). Consistent with the expression of LEF1, MMP-9 expression was reduced 1.33-fold when miR-218 mimics were transfected into LN229 cells and was significantly increased when U87 cells were transfected with miR-218 inhibitor compared to negative controls. A luciferase reporter assay further confirmed the direct interaction between miR-218 and the 3' UTR of LEF1 mRNA (Fig. 3E). The luciferase activity for the wild-type 3' UTR of LEF1 was significantly inhibited by co-transfection with miR-218 mimics compared to constructs containing mutated $3^{\prime}$ UTRs (LN229, P=0.000; U87, $\mathrm{P}=0.000)$. This experiment demonstrated that $\mathrm{LEF} 1$ is a direct target of miR-218.

In addition, we also detected the protein levels of the tissue inhibitor of metalloproteinase-1 (TIMP-1, an endogenous inhibitor of MMP-9) (20) and found no marked difference between either TIMP-1 and miR-218 or TIMP-1 and LEF1. These data suggest that miR-218 plays a critical role in GBM cell inva- 

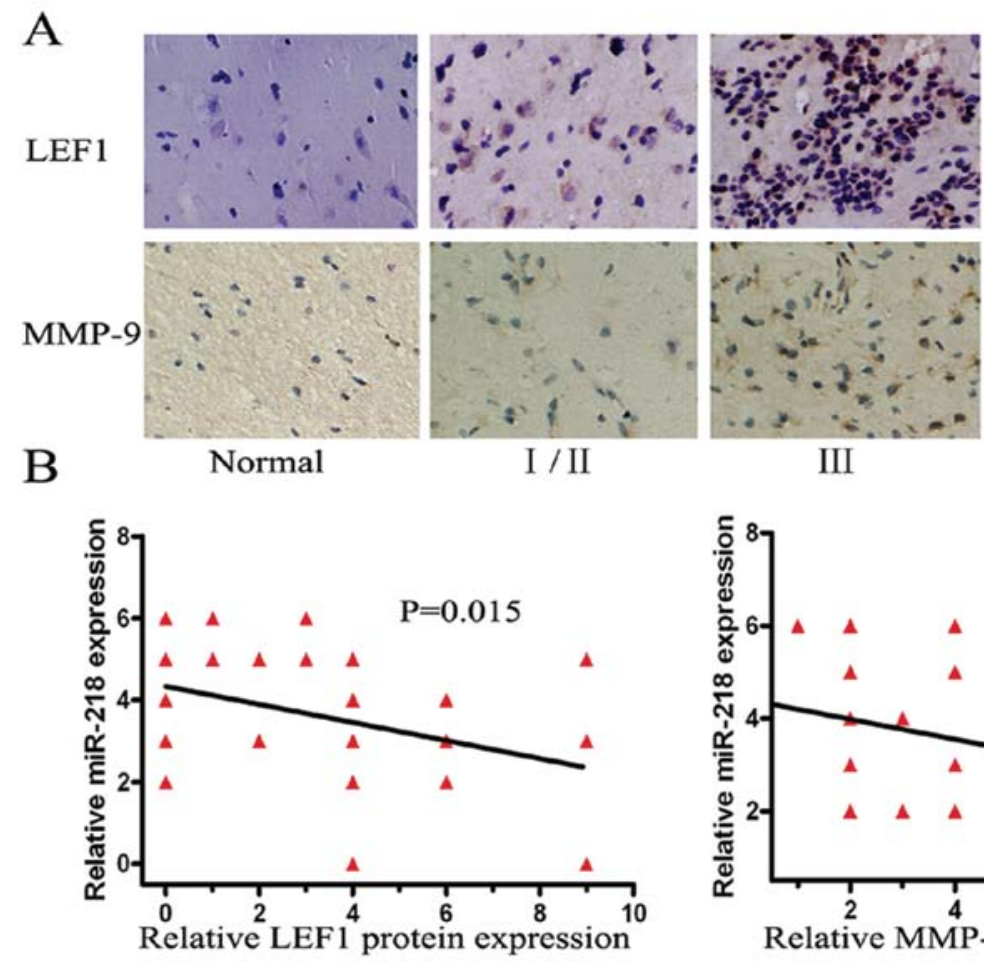

III

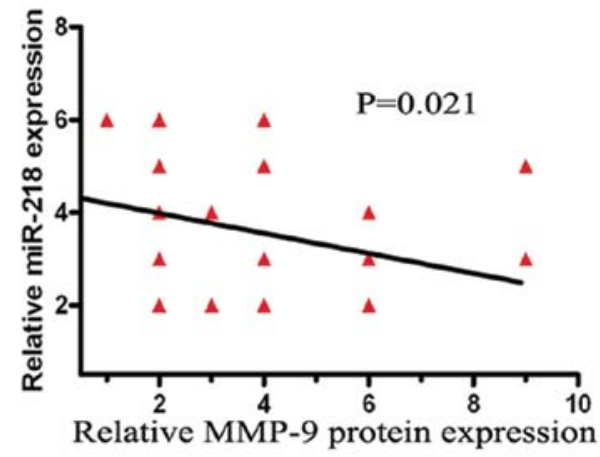

C

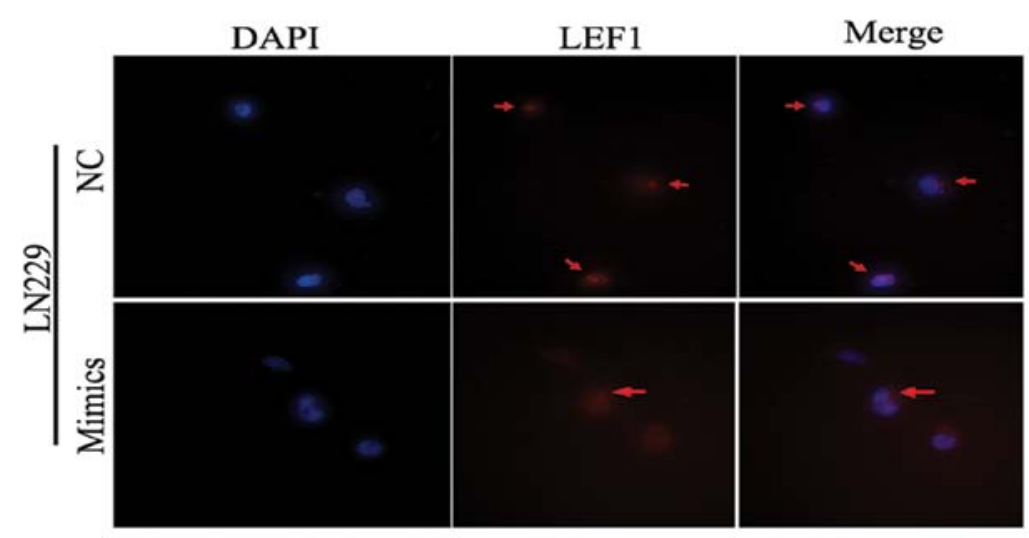

Figure 4. The inverse relationship between miR-218 and the protein levels of LEF1 and MMP-9 in the 37 tissues, and MiR-218 downregulation of LEF1 protein in LN229 cells. (A) Immunohistochemistry to detect the protein levels of LEF1 and MMP-9 in 33 different grade glioma tissues and 4 normal brain tissues. LEF1 is mainly located in the cell nucleus whereas MMP-9 is located in the cytoplasm or is extracellular. (B) An inverse relationship is depicted by the scatter diagram. (C) Immune fluorescence was performed using the highest expressed cell lines of LEF1 protein, LN229. Reactivities were visualized using the TRITC labeled ant-LEF1 (red) and nuclei were stained with DAPI (blue). The LEF1 protein signals are indicated by the red arrows.

sion by directly targeting and downregulating LEF1, thereby decreasing MMP-9 expression, but not through TIMP-1.

The protein levels of LEF1 and MMP-9 are low and inversely related to miR-218 expression in glioma tissues and miR-218 targets LEF1 $m R N A$ resulting in downregulating LEF1 protein in LN229 cells. Biological functions are ultimately carried out by a diversity of functional proteins in vivo. Therefore, we detected LEF1 and MM-9 protein levels in the aforementioned 38 tissues, including one GBM tissue which was damaged during antigen retrieval, by IHC. Both proteins were at high levels in high grade gliomas and at low levels in low grade gliomas or normal brain tissue (Fig. 4A). The degree of staining was graded by a previously described scoring system. The inverse relationship between miR-218 and LEF1 $(\mathrm{P}=0.021)$ or MMP-9 $(\mathrm{P}=0.015)$ was confirmed in these tissues by IHC and FISH (Fig. 4B). Additionally, immune fluorescence was performed using the highest expression cell lines of LEF1 protein, LN229. The signal intensity of LEF1 was markedly decreased when miR-218 was transfected into LN229 cells (Fig. 4C). As a result, the biological transport axis, miR-218-LEF1-MMP-9, was demonstrated in the invasive pathway.

LEF1 siRNA can imitate the role of miR-218 in U87. To further verify this axis, LEF1 siRNA was constructed to imitate the functions of miR-218. It was co-transfected into U87 cells with the miR-218 inhibitor. As expected, the upregulation of MMP-9 protein due to knockdown of miR-218 was significantly reduced by the specific LEF1 siRNA (Fig. 5A). This result was corroborated using the transwell invasion assay. The enhanced invasive 


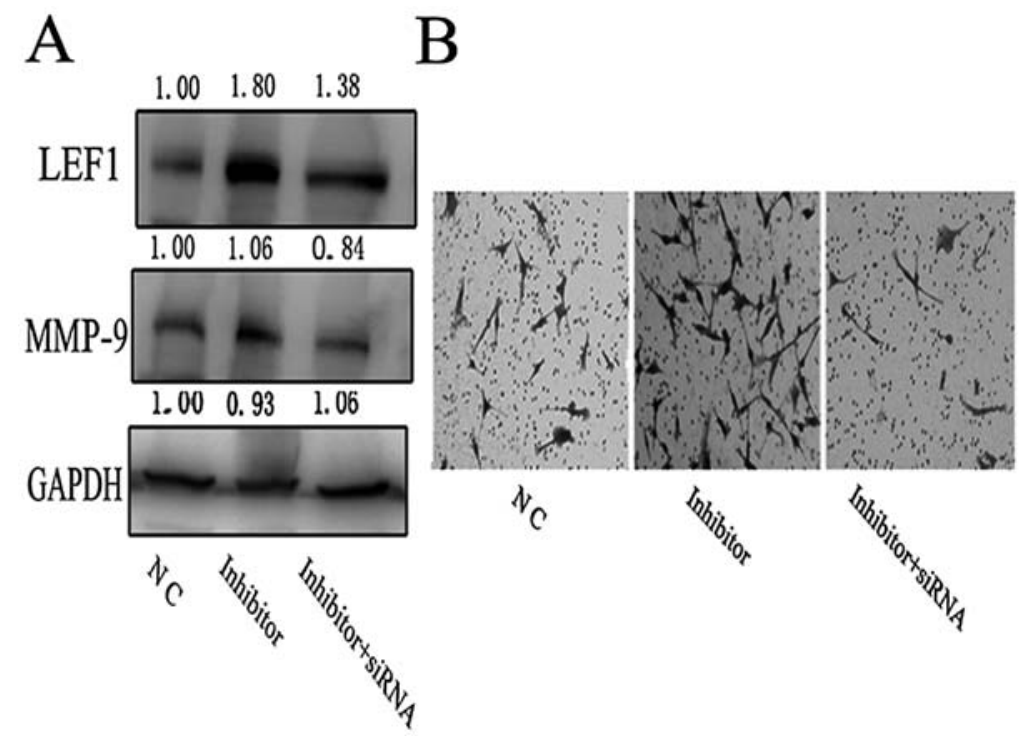

Figure 5. LEF1 siRNA can imitate the role of miR-218 in U87 cells. (B) The recovery assay was conducted using LEF1 siRNA. In the U87 cell line, LEF1 siRNA decreased the miR-218 inhibitor-induced increase in LEF1 and MMP-9 protein levels and rescued miR-218 inhibitor-induced enhanced invasive ability.

ability of miR-218 inhibitor-transfected U87 cells declined 4-fold when LEF1 siRNA was co-transfected with miR-218 inhibitor (Fig. 5B).

\section{Discussion}

GBM is the most common malignant neoplasm of the human brain. Despite advances in treatment strategies in recent years, surgery plus radiotherapy or chemotherapy, which has been used for decades, is still commonly prescribed. Combined treatment of radiotherapy plus temozolomide resulted in a slight survival rate improvement from 12.1 to 14.6 months (1). The search for new treatment methods is an urgent clinical challenge for neuroscientists. Recurrence is the most difficult problem in the treatment of GBM. Diffuse infiltration of normal peripheral tissue is a major obstacle to deciding the extent of resection. Therefore, inhibiting tumor cell invasion by blocking the invasive pathway may be beneficial in treating GBM. There are several signaling pathways that contribute to GBM pathogenesis. The AKT pathway contributes to tumor cell proliferation and apoptosis (21), the $\mathrm{NF}-\kappa \mathrm{B}$ pathway is involved in cell survival, inflammation and immune regulation, while the MAPK/ERK pathway is involved in growth and differentiation. The Notch, Toll-like receptor and TGF- $\beta$ signaling pathways also play roles. All these pathways interact to form a biological network regulating a variety of biological behavior participating in tumorigenesis. Stepwise accumulation of genetic alterations in these pathways results in tumor development. Recent reports show that aberrant expression of miRNAs contributes to many human tumors: miR-199a/b-3p in hepatocellular carcinoma (22), miR-301 in breast cancer (23), miR-99 family in prostate cancer (24), miR-200a in meningiomas (25) and miR-21 in glioblastoma (26). MiRNAs are endogenous non-coding RNAs that bind to the 3' UTR of target mRNAs to suppress translation or induce mRNA degradation. These miRNAs can regulate multiple signaling molecules belonging to different signaling pathways.
In this study, we found that miR-218 can directly target LEF1 to regulate MMP-9 expression in the Wnt pathway. Published reports and our previous studies have demonstrated that the Wnt signaling pathway significantly correlated with the invasion and proliferation of tumor cells (27). Wnts are a family of secreted glycoproteins that signal by binding the Frizzled family of receptors. This activates the modular protein disheveled, resulting in the accumulation of cytosolic $\beta$-catenin and subsequent formation of the $\beta$-catenin/ LEF1 complex in the nucleus. This DNA binding complex activates a large number of downstream target genes (including MMP-7, 9, Axin-2, cyclin D1 and Myc) $(28,29)$ causing invasion, migration, adhesion and proliferation. Dysregulation of Wnt $/ \beta$-catenin signaling was identified for the initiation of colorectal cancer development (30), but its involvement in gliomas it is not currently clear. LEF-1, a member of the LEF1/TCF transcription factor family involved in the Wnt pathway, is a DNA binding transcription factor that functions by recruiting $\beta$-catenin to Wnt target genes for regulation. It has recently been reported that levels of LEF1 are markedly correlated with tumor cell invasion and patient prognosis (31-34). Certain members of the MMP family (MMP-2, -7, -9 and -26) have been identified as downstream target-genes of the LEF1/ TCF complex. Moreover, in silico analysis revealed 4 putative LEF1/TCF binding sites in the MMP-9 proximal promoter (8).

MiR-218 is significantly downregulated and plays a critical role in the progression of many human cancers as a tumor suppressor $(5,6,15)$. The expression of miR-218 is also downregulated in gliomas but is specifically expressed or greatly enriched in normal brain tissue (35). We found a significant inverse relationship between miR-218 expression and expression of some members of the MMP family, including MMP-2, -7 and -9 in 60 GBM tissues. A single miRNA can target multiple transcripts, named a 'targetome', to regulate gene expression (36). ROBO1, BIRC5 and GJA1 in the SLIT-ROBO pathway (6), and ECOP and IKK- $\beta$ in the NF- $\kappa$ B pathway (37) were identified as direct targets of miR-218 in the regulation of cell proliferation and invasion. In this study, LEF1 was identi- 
fied as an important new target of miR-218 in the conventional prediction website (http://www.microrna.org). We detected the expression levels of miR-218, LEF1 and MMP-9 in 38 tissues, consisting of normal brain tissue, low and high grade glioma tissues by FISH and IHC and found the expression of miR-218 was always inverse to that of LEF1 $(\mathrm{P}=0.021)$ and MMP-9 proteins $(\mathrm{P}=0.015)$. Importantly, the LEF1 and MMP-9 protein levels were significantly decreased $48 \mathrm{~h}$ after transfection of miR-218 mimics in LN229 cells, whereas it was increased after transfection of miR-218 inhibitor. Therefore, we suggest that miR-218 can regulate the expression of MMPs by directly targeting LEF1. The interaction between miR-218 and LEF1 mRNA was confirmed by luciferase assays. Transwell assays and scratch tests showed that upregulation of miR-218 significantly suppressed the invasive ability of LN229 cells in vitro, while inhibiting miR-218 expression enhanced this ability. Moreover, LEF1 siRNA can rescue the invasive ability of the cells that was enhanced by exogenous expression of miR-218 inhibitor. Therefore, we suggest that miR-218 can suppress invasion by targeting LEF1 and indirectly regulating MMP-9 expression.

For the treatment of GBM, inhibiting any element of the miR218-LEF1-MMPs axis would be an effective strategy and would extend the recurrence periods. For example, the MMP inhibitor, $\alpha$ lipoic acid, blocked T cell migration into the spinal cord (38). In addition, RNA interference (RNAi) strategies include the use of small interfering RNAs (siRNAs) and miRNAs have the potential to selectively inhibit gene expression by blocking the translation of target mRNAs. They have been used as genetic tools in higher eukaryotes, and are one of the most promising therapeutic modalities for the future. However, delivering these RNAs to specific cells presents a significant challenge that requires traversing the circulatory system while avoiding kidney filtration, degradation by endonucleases, aggregation with serum proteins, and uptake by phagocytes (39). Dose-dependent toxicity has not been definitively determined in mammals. Grimm et al reported fatal side effects from abundant RNAi expression in the liver of adult mice (40). Moreover, non-specific delivery may cause side effects, including the activation of immune responses. The safety of the transfection reagent and the long-term efficacy need to be further explored.

The upstream steps of miRNA expression should be discussed here. By analysis of the Sanger miR database, Alajez et al found that miR-218 primary transcripts (hsa-miR-218-1 and hsa-miR-218-2) were embedded in the intronic regions of SLIT2 (4p15.31) and SLIT3 (5q35.1), respectively (6). They detected that miR-218 expression is increased concordant with SLIT2 and SLIT3 expression when cells were treated with the demethylation drug, 5-Aza-2-deoxycytidine. Narayan et al identified a high frequency of promoter hypermethylation in SLIT1, SLIT2 and SLIT3 in cervical cancer tumors (41). Thus it can be seen that downregulation of miR-218 results from SLIT gene promoter hypermethylation. A new therapeutic protocol for the treatment of GBM may be to test available demethylation drugs. However, to determine whether these genes are hypermethylated in gliomas require further research.

In conclusion, our previous experiments and this study show that miR-218 is also correlated with GBM cell proliferation; however, we have not extended these studies further because of the lack of specific reagents. We have demonstrated for the first time the existence of the miR-218-LEF1-MMPs axis and that it is involved in GBM cell invasion. MiR-218 binds to the $3^{\prime}$ UTR of LEF1, reducing the binding of LEF1 to the promoter of MMP-9, resulting in decreased expression of MMP-9 protein. Inhibiting any element of this axis may be an effective therapeutic strategy.

\section{Acknowledgements}

We wish to thank Yuling Yang for the tissue sample collection and clinical data retrieval. This study was supported by grants from the National Key Project of Science and Technology Supporting Programs of China (no. 2007BAI05B08), National Basic Research Program of China (973 Program) (no. 2011CB707804), China National Natural Scientific Found (30971136), Program for New Century Excellent Talents in University (NCET-070615), and the Natural Science Foundation of Tianjin Municipal Science and Technology Commission (10SYSYJC28800, 09JZCD17600).

\section{References}

1. Stupp R, Mason WP, van den Bent MJ, et al: Radiotherapy plus concomitant and adjuvant temozolomide for glioblastoma. N Engl J Med 352: 987-996, 2005.

2. Sameshima T, Nabeshima K, Toole BP, et al: Glioma cell extracellular matrix metalloproteinase inducer (EMMPRIN) (CD147) stimulates production of membrane-type matrix metalloproteinases and activated gelatinase $\mathrm{A}$ in co-cultures with brain-derived fibroblasts. Cancer Lett 157: 177-184, 2000.

3. Visse R and Nagase H: Matrix metalloproteinases and tissue inhibitors of metalloproteinases: structure, function, and biochemistry. Circ Res 92: 827-839, 2003.

4. Nagase H, Visse R and Murphy G: Structure and function of matrix metalloproteinases and TIMPs. Cardiovasc Res 69: 562-573, 2006.

5. Martinez I, Gardiner AS, Board KF, Monzon FA, Edwards RP and Khan SA: Human papillomavirus type 16 reduces the expression of microRNA-218 in cervical carcinoma cells. Oncogene 27: 2575-2582, 2008.

6. Alajez NM, Lenarduzzi M, Ito E, et al: MiR-218 suppresses nasopharyngeal cancer progression through downregulation of survivin and the SLIT2-ROBO1 pathway. Cancer Res 71: 2381-2391, 2011

7. Crawford HC, Fingleton BM, Rudolph-Owen LA, et al: The metalloproteinase matrilysin is a target of beta-catenin transactivation in intestinal tumors. Oncogene 18: 2883-2891, 1999.

8. Wu B, Crampton SP and Hughes CC: Wnt signaling induces matrix metalloproteinase expression and regulates $\mathrm{T}$ cell transmigration. Immunity 26: 227-239, 2007.

9. Yan W, Zhang W, Sun L, et al: Identification of MMP-9 specific microRNA expression profile as potential targets of anti-invasion therapy in glioblastoma multiforme. Brain Res 1411: 108-115, 2011.

10. Zhang J, Han L, Zhang A, et al: AKT2 expression is associated with glioma malignant progression and required for cell survival and invasion. Oncol Rep 24: 65-72, 2010.

11. Birner P, Toumangelova-Uzeir K, Natchev S and Guentchev M: STAT3 tyrosine phosphorylation influences survival in glioblastoma. J Neurooncol 100: 339-343, 2010.

12. Wang Y, Chen L, Bao Z, et al: Inhibition of STAT3 reverses alkylator resistance through modulation of the AKT and betacatenin signaling pathways. Oncol Rep 26: 1173-1180, 2011.

13. Debinski W and Gibo DM: Fos-related antigen 1 modulates malignant features of glioma cells. Mol Cancer Res 3: 237-249, 2005 .

14. Zhou X, Ren Y,Moore L, et al: Downregulation of miR-21 inhibits EGFR pathway and suppresses the growth of human glioblastoma cells independent of PTEN status. Lab Invest 90: 144-155, 2010.

15. Tie J, Pan Y, Zhao L, et al: MiR-218 inhibits invasion and metastasis of gastric cancer by targeting the Robol receptor. PLoS Genet 6: e1000879, 2010.

16. Yanaihara N, Caplen N, Bowman E, et al: Unique microRNA molecular profiles in lung cancer diagnosis and prognosis. Cancer Cell 9: 189-198, 2006. 
17. Rao SA, Santosh V and Somasundaram K: Genome-wide expression profiling identifies deregulated miRNAs in malignant astrocytoma. Mod Pathol 23: 1404-1417, 2010.

18. Silber J, Lim DA, Petritsch C, et al: miR-124 and miR-137 inhibit proliferation of glioblastoma multiforme cells and induce differentiation of brain tumor stem cells. BMC Med 6: 14, 2008.

19. Ingraham CA, Park GC, Makarenkova HP and Crossin KL: Matrix metalloproteinase (MMP)-9 induced by Wnt signaling increases the proliferation and migration of embryonic neural stem cells at low $\mathrm{O}_{2}$ levels. J Biol Chem 286: 17649-17657, 2011.

20. Collier IE, Legant W, Marmer B, et al: Diffusion of MMPs on the surface of collagen fibrils: the mobile cell surface-collagen substratum interface. PLoS One 6: e24029, 2011.

21. Uddin S, Hussain AR, Siraj AK, et al: Role of phosphatidylinositol 3'-kinase/AKT pathway in diffuse large B-cell lymphoma survival. Blood 108: 4178-4186, 2006.

22. Hou J, Lin L, Zhou W, et al: Identification of miRNomes in human liver and hepatocellular carcinoma reveals miR-199a/b-3p as therapeutic target for hepatocellular carcinoma. Cancer Cell 19: 232-243, 2011

23. Shi W, Gerster K, Alajez NM, et al: MicroRNA-301 mediates proliferation and invasion in human breast cancer. Cancer Res 71: 2926-2937, 2011

24. Sun D, Lee YS, Malhotra A, et al: miR-99 family of MicroRNAs suppresses the expression of prostate-specific antigen and prostate cancer cell proliferation. Cancer Res 71: 1313-1324, 2011.

25. Saydam O, Shen Y, Wurdinger T, et al: Downregulated microRNA$200 \mathrm{a}$ in meningiomas promotes tumor growth by reducing E-cadherin and activating the Wnt/beta-catenin signaling pathway. Mol Cell Biol 29: 5923-5940, 2009.

26. Chan JA, Krichevsky AM and Kosik KS: MicroRNA-21 is an antiapoptotic factor in human glioblastoma cells. Cancer Res 65 : 6029-6033, 2005.

27. Maiese K, Li F, Chong ZZ and Shang YC: The Wnt signaling pathway: aging gracefully as a protectionist? Pharmacol Ther 118: 58-81, 2008.

28. He TC, Sparks AB, Rago C, et al: Identification of c-MYC as a target of the APC pathway. Science 281: 1509-1512, 1998

29. Shtutman M, Zhurinsky J, Simcha I, et al: The cyclin D1 gene is a target of the beta-catenin/LEF-1 pathway. Proc Natl Acad Sci USA 96: 5522-5527, 1999.

30. Li TW, Ting JH, Yokoyama NN, Bernstein A, van de Wetering M and Waterman ML: Wnt activation and alternative promoter repression of LEF1 in colon cancer. Mol Cell Biol 26: 5284 5299,2006
31. Lin AY, Chua MS, Choi YL, et al: Comparative profiling of primary colorectal carcinomas and liver metastases identifies LEF1 as a prognostic biomarker. PLoS One 6: e16636, 2011.

32. Mei JM, Borchert GL, Donald SP and Phang JM: Matrix metalloproteinase(s) mediate(s) NO-induced dissociation of betacatenin from membrane bound E-cadherin and formation of nuclear beta-catenin/LEF-1 complex. Carcinogenesis 23: 2119$2122,2002$.

33. Rivat C, Le Floch N, Sabbah M, et al: Synergistic cooperation between the AP-1 and LEF-1 transcription factors in activation of the matrilysin promoter by the src oncogene: implications in cellular invasion. FASEB J 17: 1721-1723, 2003.

34. Kriegl L, Horst D, Reiche JA, Engel J, Kirchner T and Jung A: LEF-1 and TCF4 expression correlate inversely with survival in colorectal cancer. J Transl Med 8: 123, 2010.

35. Sempere LF, Freemantle S, Pitha-Rowe I, Moss E, Dmitrovsky E and Ambros V: Expression profiling of mammalian microRNAs uncovers a subset of brain-expressed microRNAs with possible roles in murine and human neuronal differentiation. Genome Biol 5: R13, 2004.

36. Selbach M, Schwanhausser B, Thierfelder N, Fang Z, Khanin R and Rajewsky N: Widespread changes in protein synthesis induced by microRNAs. Nature 455: 58-63, 2008.

37. Gao C, Zhang Z, Liu W, Xiao S, Gu W and Lu H: Reduced microRNA-218 expression is associated with high nuclear factor kappa B activation in gastric cancer. Cancer 116: 41-49, 2010.

38. Marracci GH, Jones RE, McKeon GP and Bourdette DN: Alpha lipoic acid inhibits $\mathrm{T}$ cell migration into the spinal cord and suppresses and treats experimental autoimmune encephalomyelitis. J Neuroimmunol 131: 104-114, 2002.

39. Singh S, Narang AS and Mahato RI: Subcellular fate and off-target effects of siRNA, shRNA, and miRNA. Pharm Res 28: 2996-3015, 2011.

40. Grimm D, Streetz KL, Jopling CL, et al: Fatality in mice due to oversaturation of cellular microRNA/short hairpin RNA pathways. Nature 441: 537-541, 2006.

41. Narayan G, Goparaju C, Arias-Pulido H, et al: Promoter hypermethylation-mediated inactivation of multiple Slit-Robo pathway genes in cervical cancer progression. Mol Cancer 5: 16, 2006. 